\title{
Laparoscopic Management of Stab Wound of Anterior Abdominal Wall: A Prospective Study
}

\author{
Ahmed A Darwish, MD; Ashraf Hegab, MD; Moheb Shoraby, MD. \\ Department of Surgery, Faculty of Medicine, Ain Shams University, Egypt.
}

Background: The anterior abdominal wall is one of the most common regions affected in penetrating stab wound injuries. Tradionally, wound exploration and exploratory laparotomy are used as a diagnostic and therapeutic modality in the management of such injuries. Recently, laparoscopy has gained popularity in the management of such cases with favorable results in carefully selected patients. The aim of the present study was to assess the role of laparoscopy in the diagnosis and management of patients with stab wounds of the anterior abdominal wall.

Patients and methods: 52 hemodynamically stable patients with stab wound of the anterior abdominal wall were included in this study at the emergency department of Ain Shams University Hospitals. Patients were selectively subjected to, physical examinations, focused abdominal sonography in trauma (FAST), computed tomography (CT), exploratory laparotomy, diagnostic and therapeutic laparoscopy.

Results: Out of 39 patients that underwent laparoscopy, the laparoscopy succeeded to rule out visceral injury in 14 patients (35.9\%) and was nontherapeutic in 5 patients (12.8\%). 17 patients (43.6\%) needed therapeutic management through laparoscopy and conversion to open laparotomy was done in 3 patients (7.7\%). One case of missed injury with laparoscopy was recorded (accuracy $97.4 \%$ ).

Conclusion: Laparoscopy is a feasible and useful modality for evaluating and managing hemodynamically stable patients with abdominal stab wounds. Increased use of laparoscopy will help to decrease the rate of negative and nontherapeutic laparotomies, thus lowering morbidity and decreasing length of hospitalization.

Key words: Laparoscopy, laparotomy, penetrating, stab, trauma, abdomen.

\section{Introduction:}

The abdomen is the most common region affected in penetrating injuries from stab wounds. For many decades, wound exploration and exploratory laparotomy have been used for the diagnosis and treatment of such injuries. Currently, exploratory laparotomy is reserved for those with hemodynamic instability. However, there is a wide variation in practice in the management of haemodynamically stable patients post penetrating injury of the anterior abdominal wall. ${ }^{1}$

Minimally invasive surgery has become increasingly utilized in all areas of surgery. Since the early 1990s, the learning curve in laparoscopy is increasing. Thus, it will not be surprising to expand its use in trauma. ${ }^{2}$

Negative or non-therapeutic laparotomy is associated with up to 5\% mortality and $20 \%$ morbidity rates. ${ }^{3}$ A reliable and consistent tool for identification of those patients with visceral injury who require a laparotomy is needed. Diagnostic laparoscopy (DL) may provide such a tool. Minimally invasive, using widely available equipment, DL also offers the advantage of allowing simultaneous therapeutic interventions. ${ }^{4}$

The aim of the present study is to assess the role of laparoscopy in the diagnosis and management of patients with penetrating stab trauma of the anterior abdominal wall. 


\section{Patients and methods:}

This prospective study was conducted over a period of 3 years from May 2012 to June 2015 at the emergency department of Ain Shams university Hospitals, Cairo, Egypt.

The study included 52 patients with single penetrating stab injury of the anterior abdominal wall who were selectively subjected to 1) Physical examination, 2) Focused abdominal sonography in trauma (FAST) with examination of four areas for free fluid: peri-hepatic and hepatorenal space, perisplenic, pelvis and pericardium, 3) computed tomography (CT) (abdomen and pelvis) to assess the presence of free fluid and solid organ injuries and 4) Local wound exploration (LWE).

Patients underwent exploratory laparotomy (EL) if they were hemodynamically unstable (persistent systolic blood pressure $<90$ $\mathrm{mmHg}$ ) or if they were hemodynamically stable with visceral evisceration through the stab wound.

If intra-abdominal fluid accumulation was detected by FAST in hemodynamically stable patients without visceral evisceration, they were also subjected to laparoscopy.

Evidence of fascial breach on LWE in patients without positive findings by FAST as well as positive findings in CT for patients with equivocal LWE test were also subjected to laparoscopy.

Otherwise, patients with no evidence of fascial breach on LWE or with negative $\mathrm{CT}$ findings were treated by non-operative regimen including simple wound repair and repeated physical assessment to exclude any signs of peritonitis that necessitated surgical intervention.

Data extracted for analysis included demographic information, causes of injury, radiological findings, operative procedures, outcomes of laparoscopy and injuries that were treated. In addition, postoperative outcomes including length of hospital stay, complications, and mortalities were assessed.

Surgical Technique: Laparoscopic exploration was performed while the patient was supine. After clamping the stab wound with towel clips, pneumoperitoneum with carbon dioxide (CO2) was established via the open Hasson technique at the umbilicus. The pressure was maintained at $12-15 \mathrm{mmHg}$. A $10-\mathrm{mm} 30^{\circ}$ laparoscope was inserted via a $10-\mathrm{mm}$ trocar into the peritoneal cavity. If there was no peritoneal perforation, the laparoscopic procedure was terminated and the abdominal wound was repaired.

In cases of peritoneal perforation, a $10-\mathrm{mm}$ camera port was created at the suprapubic region for alternative use. Additionally, two further trocars of 5-10 mm were introduced at right and left paramedian sites as working ports.

The abdominal cavity was explored systematically including solid organs, stomach, omentum, transverse colon, and diaphragm on the patient's left side from the umbilical camera port with the patient in the reverse Trendenlenberg position. The pancreas and the posterior gastric wall were inspected after the scope was directed into the lesser sac by dissecting the gastrocolic ligament if a hematoma or fluid accumulation was found in the lesser sac. After close inspection of the upper abdominal structures, the patient was placed in a steep Trendenlenberg position, which allowed inspection of the pelvic structures, including the recto-sigmoid colon, the urinary bladder, and the iliac regions.

For complete evaluation of the ascending colon and the small bowel, the telescope was then inserted into the suprapubic port, and atraumatic grasping forceps were introduced through the umbilical port and through the left paramedian trocar. Using the two forceps, the bowel was inspected from the ascending colon, ileo-ceacal valve, and to the distal two thirds of the small bowel for any mesenteric tears or perforation. The surgeon then changed his position, moving to the patient's right side to facilitate inspection of the proximal onethird of the small bowel and the descending colon utlizing the same maneuver. It was crucial to tilt the operating table right, left, up, and down for easier exposure by shifting the abdominal contents.

Once a significant intra-abdominal lesion had been documented, therapeutic 
intervention was done in the form of the following: (1) hemostasis of bleeding solid organs, or mesenteric injuries with electrocautery or suture ligation; (2) repair of diaphragmatic tear with intracorporeal sutures; and (3) repair of perforated hollow organs with laparoscopic procedures.

Laparoscopy was classified as negative if there was no injury, as nontherapeutic if there was an injury but did not require a surgical intervention, therapeutic if an injury was identified and repaired, and positive if there was an injury that required conversion to open exploration for better management.

\section{Results:}

There were 65 patients identified with single abdominal stab injury over the 3 years study period in the emergency department of Ain Shams University Hospitals. 13 patients were excluded from the study as they were hypotensive on arrival that needed resuscitation and emergency exploratory laparotomy (EL). 52 hemodynamically stable patients were included in this study, their ages ranged from 18-50 years with a mean of $28.8 \pm 8.5$ years. 49 (94.2\%) of them were males while 3 (5.8\%) were females.

The majority of injuries were caused by knives (84.6\%), and the rest were with varied instruments including clasp knives, skewers and swords.

Visceral evisceration was diagnosed in 4 patients and they underwent EL, while 48 patients had no visceral evisceration and underwent FAST examination. A FAST was positive in 13 patients who underwent laparoscopy, while it was negative in 35 patients so; LWE was done for them to detect any fascial breach. LWE was positive in 20 patients who underwent laparoscopy while it was negative in 5 patients and equivocal in 10 patients. CT was done for patients with equivocal LWE which was positive in 6 patients who underwent laparoscopy and negative in 4 patients. Patients with negative findings on LWE or CT were observed for 24 hours with serial physical examination to detect signs of peritonitis.

Out of the 39 patients who underwent laparoscopy, peritoneal breach was detected in 33 patients with concomitant visceral injury in 25 patients of them.

Laparoscopy ruled out visceral injury (negative laparoscopy) in 14/39 (35.9\%) patients with or without peritoneal breach (8 and 6 patients respectively) that needed repair of abdominal wound \pm peritoneal repair.

Non-therapeutic laparoscopy was performed in 5 patients (12.8\%): 4 patients presented with minor liver lacerations and one with a retroperitoneal hematoma related to the ascending colon with no significant colonic injury.

Therapeutic laparoscopy was performed in 17 patients (43.6\%): 2 patients with liver lacerations that needed hemostasis by electrocautery, 4 patients with stomach perforation were repaired with or without omental patch, 7 patients with bowel injuries, 2 patients with mesenteric injuries, 1 patient with a diaphragmatic injury, and 1 patient with a splenic tear that all needed laparoscopic management.

Conversion to open EL (positive laparoscopy) was done in 3 patients (7.7\%): one with a splenic tear that needed splenectomy from a left subcostal incision, one with a retroperitneal hematoma and one with a combined injury Table (1).

Peritonitis occurred in a single patient due to a missed intestinal perforation that necessitated an EL with an accuracy of the laparoscope to diagnose visceral injury of 97.4\% (38/39 patients).

Among patients who required EL, one patient had a minor complication in the form of wound infection.

The average length of hospital stay was $3 \pm 2.5$ days for all patients.

Laparoscopy was negative in 14 patients and was non-therapeutic in 5 patients. Also, 16 patients were managed successfully with laparoscopy. Overall, because of the use of laparoscopy, laparotomy was avoided in $35 / 52$ (67.3\%) of the patients in this study.

\section{Discussion:}

The evaluation of a trauma patient starts with the advanced trauma life support 
primary survey followed by a thorough physical examination. LWE is limited in its ability to determine specific intra-abdominal injuries, but it can often determine fascial penetration and thereby avoid the need for further studies. ${ }^{5}$

The usual diagnostic procedures, FAST, and even CT, all have their strengths and weaknesses and none of them are 100\% reliable. For this reason, exploratory laparotomy is often performed in the case of stab wounds, but with a high morbidity percentage that reaches up to $40 \% .{ }^{6}$ Therefore, the main benefits of laparoscopy are that it can reduce the rate of non-therapeutic and negative laparotomies, and even provide a therapeutic option. ${ }^{7}$

Different algorithms have been developed by many trauma centers worldwide for the management of PAT to aid the fast and effective diagnosis and management of visceral injuries. ${ }^{7}$ In our study, we proposed a modified algorithm for diagnosis and management of patients presented with stab injury of the anterior abdominal wall Figure (1).

Recent literature suggested that the role of laparoscopy in trauma was not fully established. However; with accumulated experience in laparoscopic surgery and the advent of new instrumentation, laparoscopic surgery has gained acceptance in intraabdominal operations as well as in the trauma setting. The role of laparoscopy in trauma thus is continually evolving. 4

For trauma patients, laparoscopy provides clear visualization of the peritoneal space and anterior abdominal wall, and unlike other diagnostic modalities, has the additional benefit of potential for therapeutic intervention. ${ }^{5}$

The first reports demonstrating the utility of laparoscopic surgery in the evaluation of trauma patients were published in the 1920s which investigated its use as a method to diagnose internal bleeding in patients with PAT. 8,9

The present study adds to the growing data indicating that laparoscopy can play a major role in the evaluation and management of stable patients with penetrating trauma to the abdomen. ${ }^{10,11,12}$

The main aim of our study is to assess the role of laparoscopy in: (1) Reducing nontherapeutic laparotomy; (2) Avoiding missed injuries; and (3) Maximizing therapeutic laparoscopy.

Villavicencio and Aucar ${ }^{10}$ authored an extensive review in 1997 in which they compared outcomes collected from 37 separate studies, involving over 1900 patients, they found that $63 \%$ of patients who underwent laparoscopic evaluation avoided laparotomy. When comparing DL and EL in abdominal stab wounds, the results showed that DL reduced the nontherapeutic laparotomy rate from $65 \%$ to $11 \%$ (lowest) or $50 \%$ (highest). Besides, DL reduced unnecessary laparotomies (calculated as $100 \%$ conversion to laparotomy rate) in 55$87 \%$ of trauma cases.

In 1976, Gazzangia et al. ${ }^{13}$ evaluated 37 patients; in 14 of these patients, laparotomy was avoided because of a negative diagnostic laparoscopy (DL). There were no falsenegative investigations. They concluded that the use of diagnostic laparoscopy in abdominal trauma was useful to decrease the rate of negative laparotomy.

In the largest study on laparoscopy in PT, Zantut et al., ${ }^{14}$ reported a multicenter retrospective study of 510 hemodynamically stable patients who underwent DL for PT. The inclusion criterion for the study was a hemodynamically stable patient who had penetration of the anterior fascia by a stab wound or a gunshot wound with a possible intraperitoneal injury. Negative or nontherapeutic laparotomy was avoided in 303 (59.4\%) patients, of whom 26 patients received a therapeutic laparoscopic intervention.

The results of our study showed that the laparoscopy-based strategy to reduce the nontherapeutic laparotomy had succeeded to decrease the rate from $35.8 \%$ to $0 \%$ and prevented laparotomies in $89.7 \%$ of trauma cases.

The use of laparoscopy as a diagnostic and therapeutic tool led to avoidance of an open 


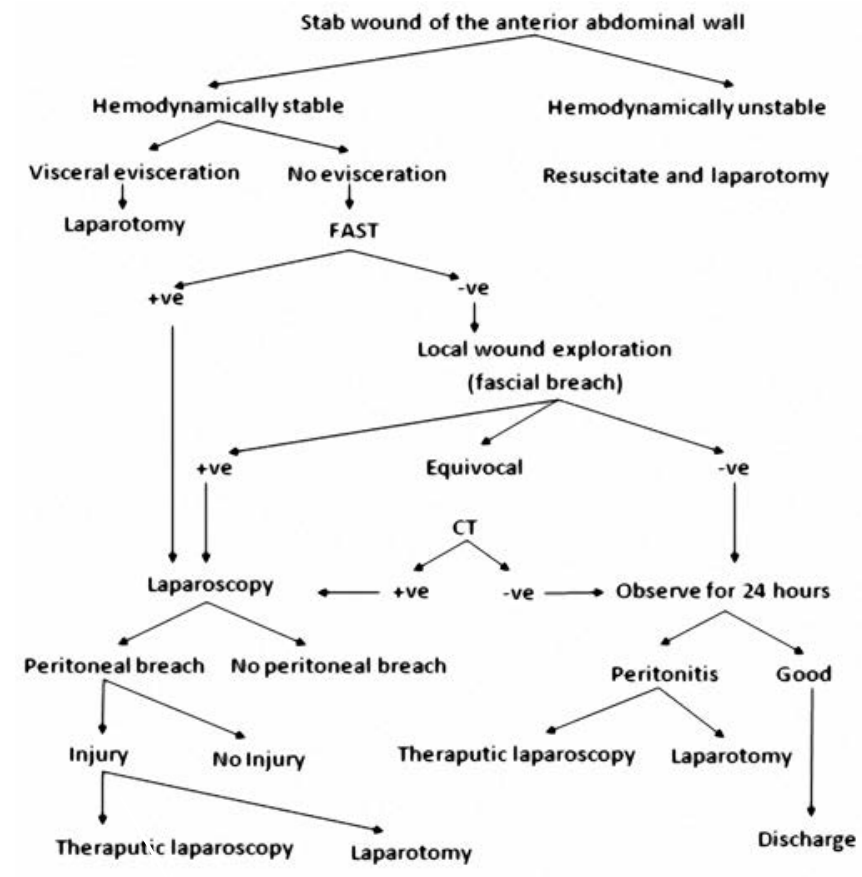

Figure (1): Diagnosis and treatment algorithm for stab injury of anterior abdominal wall.

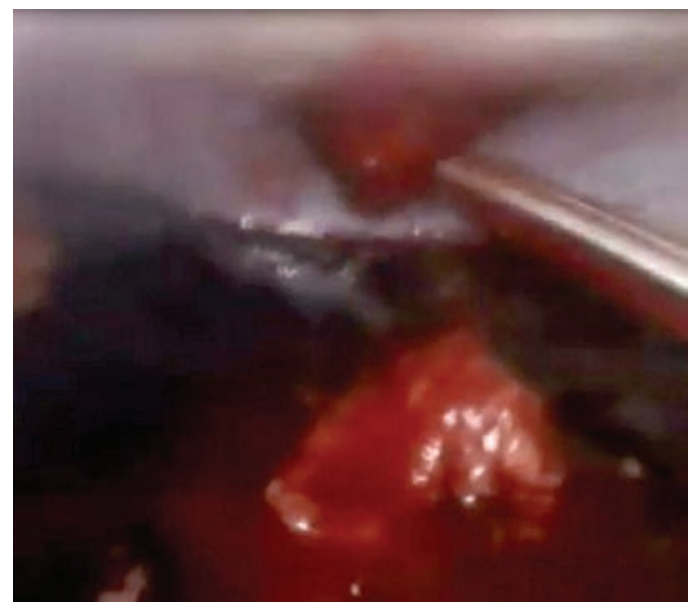

Figure (2): Site of peritoneal penetration.

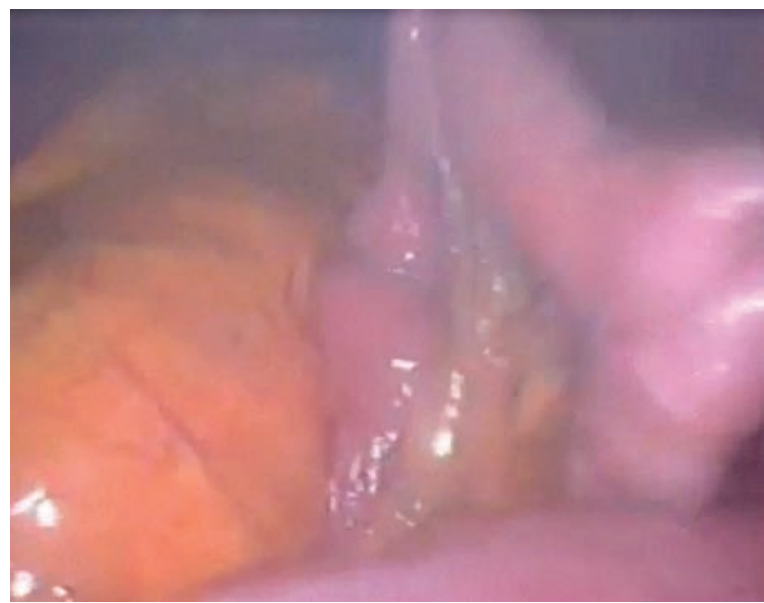

Figure (3): Small bowel exploration.

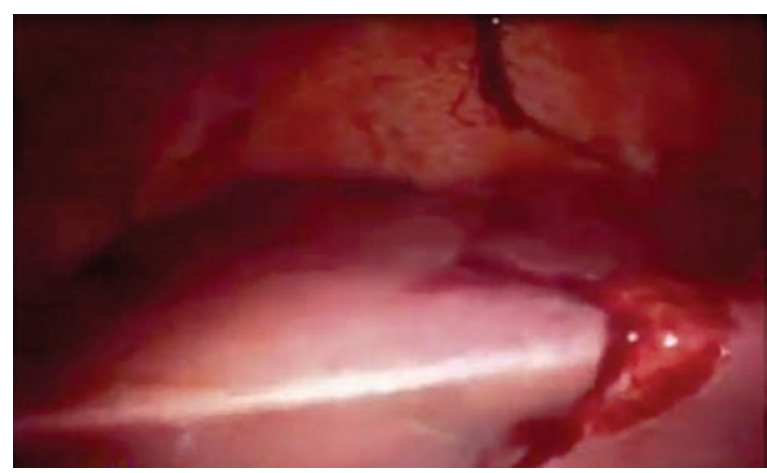

Figure (4): Liver tear without active bleading.

surgery in more than $67 \%$ (35/52) of patients in our study.

A major drawback of utilizing DL for abdominal stab wounds is missed injuries.
Visualization of solid organs is simple to perform and reliable, but complete examination of the intestines presents a greater challenge for surgeons with a reported 

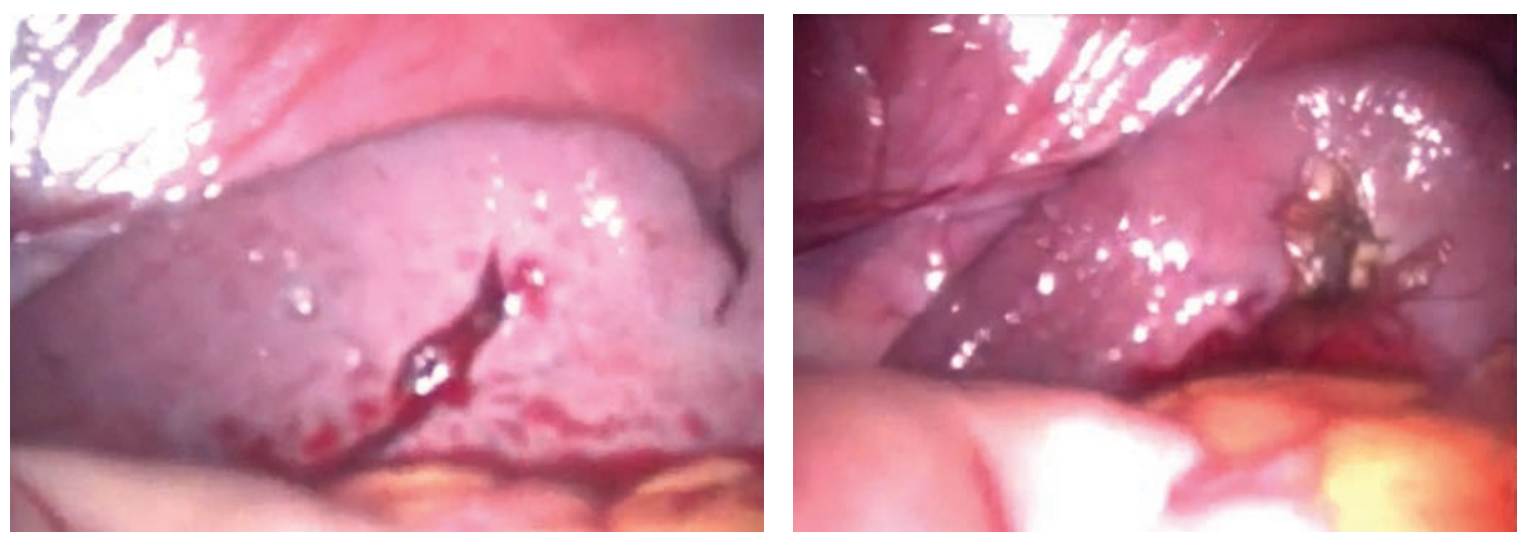

Figure (5): Splenic tear before and after cauterization.

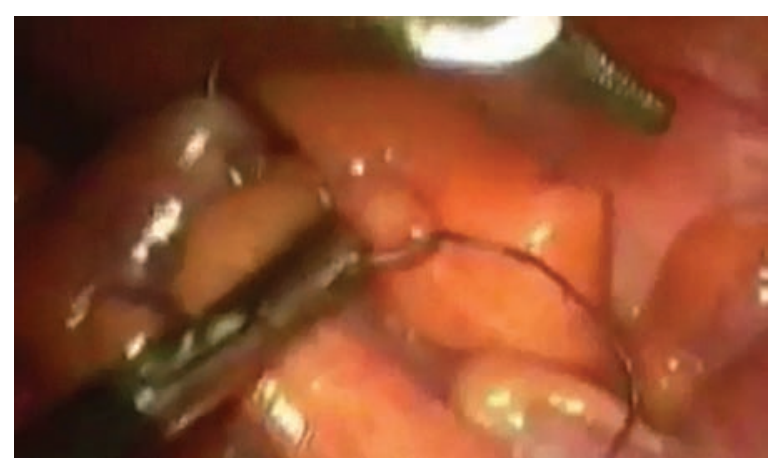

Figure (6): Small intestinal perforation suturing with omental flap.

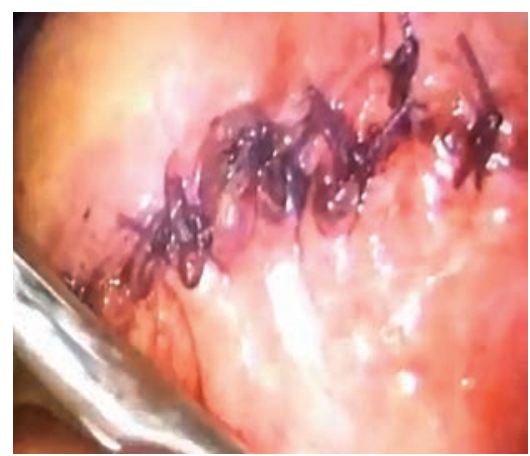

Figure (7): Repair of the Rt. colon.

Table (1): Laparoscopic findings and managements of patients with penetrating abdominal injuries:

\begin{tabular}{|l|l|l|}
\hline \multicolumn{1}{|c|}{ Finding at laparoscopy } & No (out of 39) & \multicolumn{1}{c|}{ Surgical procedure } \\
\hline No injury & 14 & Diagnostic laparoscopy \\
\hline Liver tear & 6 & $\begin{array}{l}\text { Non therapeutic laparoscopy (n=4) and } \\
\text { laparoscopic hemostasis (n=2) }\end{array}$ \\
\hline Ant. gastric perforation & 4 & Laparoscopic repair \\
\hline Small intestinal perforation & 4 & Laparoscopic repair \\
\hline Colonic injury & $3(2 \mathrm{Rt} \&$ 1 Lt) & Laparoscopic repair \\
\hline Mesenteric injury & 2 & Laparoscopic hemostasis \\
\hline Splenic tear & 2 & $\begin{array}{l}\text { Laparoscopic hemostasis (n=1) and open } \\
\text { splenectomy (n=1) }\end{array}$ \\
\hline Retroperitoneal hematoma & 2 & $\begin{array}{l}\text { Non therapeutic laparoscopy (n=1) and } \\
\text { exploratory laparotomy (n=1) }\end{array}$ \\
\hline Diaphragm & 1 & Laparoscopic repair \\
\hline Combined injury & 1 & Exploratory laparotomy \\
\hline
\end{tabular}

missed injury rate of $40 \% .^{2,15}$

The key points to avoid missed injuries in DL include the following: (1) a systematic and careful inspection; (2) changing the patient's position during laparoscopic procedures for inspection; and (3) use of atraumatic grasping forceps for bowel manipulation. Nevertheless, we should not hesitate to convert DL to laparotomy if complete visualization of the intra-abdominal structures cannot be obtained. 
Table (2): Patient characteristics and outcomes:

\begin{tabular}{|l|l|}
\hline \multicolumn{1}{|c|}{ Patient characteristics } & No (out of 52) \\
\hline Male & 49 \\
\hline Female & 3 \\
\hline Age & $18-50$ years \\
\hline Laparotomy from start & 4 \\
\hline Negative laparoscopy & 14 \\
\hline Nontheraputic laparoscopy & 5 \\
\hline Theraputic laparoscopy & 17 \\
\hline Positive laparoscopy & 3 \\
\hline Conservative & 9 \\
\hline Length of hospital stay & $3 \pm 2.5$ days \\
\hline Complications & 2 \\
\hline Mortality & 0 \\
\hline
\end{tabular}

Despite optimum strategy not to miss an injury, a case of missed intestinal perforation was documented in this study probably because it was a small perforation at the mesenteric border of a jujenal loop with an accuracy of laparoscopy to diagnose visceral injury of $97.4 \%$ (38 out of 39 patients).

Althoughseveralpreviouslypublishedtrials have reported on the laparoscopic treatment of certain injuries in hemodynamically stable abdominal trauma patients, the role of laparoscopy as a therapeutic tool for PAT is still uncertain. ${ }^{14,16,17}$

Chol and Lim used laparoscopy to treat bowel perforations by totally laparoscopic, laparoscopically-assisted, and handassisted procedures with a high success rate. Treating patients with injuries such as bowel perforations is technically demanding. 18

In the present study, we attempted to apply laparoscopically based procedures for the hemodynamically stable abdominal stab wound patients. Significant intra-abdominal injuries were identified and treated by laparoscopic procedures in 17 patients: 2 liver lacerations needed hemostasis by electrocautery, 4 stomach perforations, 7 bowel injuries, 2 mesenteric injuries, 1 diaphragmatic injury, and 1 splenic tear that all needed laparoscopic management.

However, conversion to laparotomy was done in 3 patients: one splenic tear that needed splenectomy by left subcostal incision, one retroperitneal hematoma and one combined injury.

With respect to outcomes, most authors report a decreased complication rate, shorter length of hospital stay, and decreased costs when negative laparoscopy is compared with a negative or nontherapeutic laparotomy. 14,19,20

The results in the present study, like most other studies, show that nontherapeutic laparoscopy is lesser invasive than nontherapeutic laparotomy with short length of hospital stay and less postoperative morbidity and mortality.

Laparoscopy in the trauma setting has been associated with some complications including tension pneumothorax, raised intracranial pressure and gas embolism. ${ }^{21}$ Although Small numbers of such complications were reported in the 1990s, none was detected in this study as they now seem to be preventable if suitable measures are adopted.

\section{Conclusion:}

Current trends in all areas of surgery are towards less invasive techniques, laparoscopy is feasible and useful modality for evaluating and managing hemodynamically stable patients with abdominal stab wounds. Increased use of laparoscopy will help to decrease the rate of negative and nontherapeutic laparotomies, 
thus lowering morbidity, decreasing length of hospitalization.

\section{Reference:}

1- Mitra B, Gocentas R, O’Reilly G, Cameron PA, Atkin C: Management of haemodynamically stable patients with abdominal stab wounds. Emergency Medicine Australasia 2007; 19: 269-275.

2- Fabian TC, Croce MA, Stewart RM, Pritchard FE, Minard G, Kudsk KA: A prospective analysis of diagnostic laparoscopy in trauma. Ann Surg 1993; 217: 557-565.

3- Shih HC, Wen YS, Ko TJ, Wu JK, Su CH, Lee CH: Noninvasive evaluation of blunt abdominal trauma: Prospective study using algorithms to minimise nontherapeutic laparotomy. World Surg 1999; 123: 263-270.

4- O’Malley E, Boyle E, O’Callaghan A, Coffey JC, Walsh SR: Role of laparoscopy in penetrating abdominal trauma: A systematic review. World J Surg 2013; 37: 113-122.

5- Miles EJ, Dunn E, Howard D, Mangram A: The role of laparoscopy in penetrating abdominal trauma. JSLS 2004; 8: 304-309.

6- Leppäniemi A, Salo J, Haapiainen R: Complications of negative laparotomy for truncal stab wounds. J Trauma 1995; 38: 54-58.

7- Uranüs S, Dorr K: Laparoscopy in abdominal trauma. Eur J Trauma Emerg Surg 2010; 36: 19-24.

8- Short AR: The uses of celioscopy. BMJ 1925; 2: 254-255.

9- Stone WE: Intra-abdominal examination by the aid of the peritoneoscope. $J$ Kansas Med Soc 1924; 24: 63-65.

10- Villavicencio RT, Aucar JA: Analysis of laparoscopy in trauma. J Am Coll Surg 1999; 189: 11-20.

11- Ivatury RR, Simon RJ, Stahl WM: Selective celiotomy for missile wounds of the abdomen based on laparoscopy. Surg Endosc 1994; 8:
366-370.

12- Matthews BD, Bui H, Harold KL, Kercher KW, Adrales G, Park A, et al: Laparoscopic repair of traumatic diaphragmatic injuries. Surg Endosc 2003; 17: 254-258.

13- Gazzaniga AB, Stanton WW, Bartlett RH: Laparoscopy in the diagnosis of blunt and penetrating injuries to abdomen. Am J Surg 1976; 131: 315-318.

14- Zantut LF, Ivatury RR, Smith RS, Kawahara NT, Porter JM, Fry WR, et al: Diagnostic and therapeutic laparoscopy for penetrating abdominal trauma: A multicenter experience. J Trauma 1997; 42: 825-831.

15- Rossi P, Mullins D, Thal E: Role of laparoscopy in the evaluation of abdominal trauma. Am J Surg 1993; 166: 707-711.

16- Chen RJ, Fang JF, Lin BC, Hsu YB, Kao $\mathrm{JL}$, Kao YC, et al: Selective application of laparoscopy and fibrin glue in the failure of nonoperative management of blunt hepatic trauma. J Trauma 1998; 44: 691-695.

17- Kawahara NT, Alster C, Fujimura I, Poggetti RS, Biroloni D: Standard examination system for laparoscopy in penetrating abdominal trauma. J Trauma 2009; 67: 589-595

18- Chol YB, Lim KS: Therapeutic laparoscopy for abdominal trauma. Surg Endosc 2003; 17: 421-427.

19- Hallfeldt KK, Trupka AW, Erhard J, Waldner $\mathrm{H}$, Schweiberer L: Emergency laparoscopy for abdominal stab wounds. Surg Endosc 1998; 12: 907-910.

20- DeMaria EJ, Dalton JM, Gore DC, Kellum JM, Sugerman HJ: Complementary roles of laparoscopic abdominal exploration and diagnostic peritoneal lavage for evaluating abdominal stab wounds: A prospective study. J Laparoendosc Adv Surg Tech A 2000; 10 : 131-136.

21- Leppäniemi A, Haapiainen R: Diagnostic laparoscopy in abdominal stab wounds: A prospective, randomized study. $J$ Trauma 2003; 55: 636-645. 